\title{
ANISOTROPIC GEODESIC FLUID SPHERES IN GENERAL RELATIVITY.
}

\author{
L. Herrera* \\ Escuela de Física, Facultad de Ciencias, \\ Universidad Central de Venezuela, \\ Caracas, Venezuela. \\ J. Martin ${ }^{\dagger}$ and J. Ospino \\ Area de Física Teórica. Facultad de Ciencias. \\ Universidad de Salamanca. 37008 Salamanca, España.
}

\begin{abstract}
It is shown that unlike the perfect fluid case, anisotropic fluids (principal stresses unequal) may be geodesic, without this implying the vanishing of (spatial) pressure gradients. Then the condition of vanishing four acceleration is integrated in non-comoving coordinates. The resulting models are necessarily dynamic, and the mass function is expressed in terms of the fluid velocity as measured by a locally Minkowskian observer. An explicit example is worked out.
\end{abstract}

${ }^{*}$ Postal address: Apartado 80793, Caracas 1080 A, Venezuela; e-mail address: laherrera@telcet.net.ve

†e-mail address:chmm@usal.es 


\section{Introduction}

As is well known, the vanishing of four acceleration (geodesic condition) implies for a perfect fluid that pressure gradients vanish. In the case of spherical bounded ( non-dissipative) configuration, the vanishing of pressure at the boundary surface, implies in turn the vanishing of pressure everywhere within the distribution (dust).

Indeed, for a perfect fluid the equation of motion reads

$$
(\rho+p) a^{\alpha}=h^{\alpha \nu} p_{, \nu}
$$

with

$$
\begin{gathered}
h_{\mu}^{\alpha} \equiv \delta_{\mu}^{\alpha}-u^{\alpha} u_{\mu} \\
a^{\mu}=u^{\nu} u_{; \nu}^{\mu}
\end{gathered}
$$

where colon and semicolon denote partial and covariant derivatives, and as usual $a^{\mu}, u^{\mu}, \rho$ and $p$ stand for the four acceleration, the four velocity, the energy density and the pressure respectively.

¿From the above it becomes evident that the geodesic condition implies the vanishing of pressure gradients. From purely physics considerations this conclusion is also obvious: the vanishing of four-acceleration means that only gravitational forces are acting on any fluid element, thereby implying that pressure gradients (the only hydrodynamic force in a perfect fluid) vanish. However in the case of anisotropic fluids, an additional force term appears besides the pressure gradient (see next section). Therefore it is in principle possible to have a fluid distribution, such that both terms cancel each other, leading to a geodesic fluid with non-vanishing pressure gradients.

Since the original Lemaitre paper [1] and particularly since the work of Bowers and Liang [2] anisotropic fluids have attracted the attention of many researchers in relativity and relativistic astrophysics (see [3] and references therein), due to the conspicuous role played by local anisotropy of pressure in the structure and evolution of self-gravitating objects. It is the purpose of this work to present further models of anisotropic spheres, based on the geodesic condition. Besides the natural interest of such models in general relativity, the presented models are interesting because they represent the generalization of Tolman-Bondi [4] models to anisotropic fluids, in non-comoving coordinates. Incidentally, it is worth noticing that in the classical paper by Oppenheimer and Snyder on dust collapse [5], they start their study, using 
the same kind of coordinates we use here, and then switch to comoving ones, in order to integrate the field equations.

The plan of the paper is as follows. In Section 2 we define the conventions and give the field equations and expressions for the kinematic variables we shall need, in noncomoving coordinates. The geodesic condition is explicitly integrated in Section 3. In Section 4 we work out an example. Finally a discussion of results is presented in Section 5.

\section{Relevant Equations and Conventions}

We consider spherically symmetric distributions of collapsing anisotropic fluid, which we assume to evolve adiabatically (without dissipation), bounded by a spherical surface $\Sigma$.

The line element is given in Schwarzschild-like coordinates by

$$
d s^{2}=e^{\nu} d t^{2}-e^{\lambda} d r^{2}-r^{2}\left(d \theta^{2}+\sin ^{2} \theta d \phi^{2}\right)
$$

where $\nu(t, r)$ and $\lambda(t, r)$ are functions of their arguments. We number the coordinates: $x^{0}=t ; x^{1}=r ; x^{2}=\theta ; x^{3}=\phi$.

The metric (4) has to satisfy Einstein field equations

$$
G_{\mu}^{\nu}=-8 \pi T_{\mu}^{\nu}
$$

which in our case read [6]:

$$
\begin{aligned}
-8 \pi T_{0}^{0} & =-\frac{1}{r^{2}}+e^{-\lambda}\left(\frac{1}{r^{2}}-\frac{\lambda^{\prime}}{r}\right), \\
-8 \pi T_{1}^{1} & =-\frac{1}{r^{2}}+e^{-\lambda}\left(\frac{1}{r^{2}}+\frac{\nu^{\prime}}{r}\right), \\
-8 \pi T_{2}^{2}=-8 \pi T_{3}^{3}=- & \frac{e^{-\nu}}{4}(2 \ddot{\lambda}+\dot{\lambda}(\dot{\lambda}-\dot{\nu})) \\
& +\frac{e^{-\lambda}}{4}\left(2 \nu^{\prime \prime}+\nu^{\prime 2}-\lambda^{\prime} \nu^{\prime}+2 \frac{\nu^{\prime}-\lambda^{\prime}}{r}\right), \\
& -8 \pi T_{01}=-\frac{\dot{\lambda}}{r}
\end{aligned}
$$


where dots and primes stand for partial differentiation with respect to $t$ and $r$, respectively.

In order to give physical significance to the $T_{\nu}^{\mu}$ components we apply the Bondi approach [6].

Thus, following Bondi, let us introduce purely locally Minkowski coordinates $(\tau, x, y, z)$ (alternatively one may introduce a tetrad field associated to locally Minkowskian observers).

$$
d \tau=e^{\nu / 2} d t ; \quad d x=e^{\lambda / 2} d r ; \quad d y=r d \theta ; \quad d z=r \sin \theta d \phi .
$$

Then, denoting the Minkowski components of the energy tensor by a bar, we have

$\bar{T}_{0}^{0}=T_{0}^{0} ; \quad \bar{T}_{1}^{1}=T_{1}^{1} ; \quad \bar{T}_{2}^{2}=T_{2}^{2} ; \quad \bar{T}_{3}^{3}=T_{3}^{3} ; \quad \bar{T}_{01}=e^{-(\nu+\lambda) / 2} T_{01}$.

Next, we suppose that when viewed by an observer moving relative to these coordinates with proper velocity $\omega(t, r)$ in the radial direction, the physical content of space consists of an anisotropic fluid of energy density $\rho$, radial pressure $P_{r}$ and tangential pressure $P_{\perp}$. Thus, when viewed by this moving observer the covariant tensor in Minkowski coordinates is

$$
\left(\begin{array}{cccc}
\rho & 0 & 0 & 0 \\
0 & P_{r} & 0 & 0 \\
0 & 0 & P_{\perp} & 0 \\
0 & 0 & 0 & P_{\perp}
\end{array}\right) .
$$

Then a Lorentz transformation readily shows that

$$
\begin{gathered}
T_{0}^{0}=\bar{T}_{0}^{0}=\frac{\rho+P_{r} \omega^{2}}{1-\omega^{2}}, \\
T_{1}^{1}=\bar{T}_{1}^{1}=-\frac{P_{r}+\rho \omega^{2}}{1-\omega^{2}}, \\
T_{2}^{2}=T_{3}^{3}=\bar{T}_{2}^{2}=\bar{T}_{3}^{3}=-P_{\perp}, \\
T_{01}=e^{(\nu+\lambda) / 2} \bar{T}_{01}=-\frac{\left(\rho+P_{r}\right) \omega e^{(\nu+\lambda) / 2}}{1-\omega^{2}},
\end{gathered}
$$


Note that the coordinate velocity in the $(t, r, \theta, \phi)$ system, $d r / d t$, is related to $\omega$ by

$$
\omega(t, r)=\frac{d r}{d t} e^{(\lambda-\nu) / 2} .
$$

Feeding back (10 13) into (6 9), we get the field equations in the form

$$
\begin{gathered}
\frac{\rho+P_{r} \omega^{2}}{1-\omega^{2}}=-\frac{1}{8 \pi}\left\{-\frac{1}{r^{2}}+e^{-\lambda}\left(\frac{1}{r^{2}}-\frac{\lambda^{\prime}}{r}\right)\right\}, \\
\frac{P_{r}+\rho \omega^{2}}{1-\omega^{2}}=-\frac{1}{8 \pi}\left\{\frac{1}{r^{2}}-e^{-\lambda}\left(\frac{1}{r^{2}}+\frac{\nu^{\prime}}{r}\right)\right\}, \\
P_{\perp}=-\frac{1}{8 \pi}\left\{\frac{e^{-\nu}}{4}(2 \ddot{\lambda}+\dot{\lambda}(\dot{\lambda}-\dot{\nu}))\right. \\
\left.-\frac{e^{-\lambda}}{4}\left(2 \nu^{\prime \prime}+\nu^{\prime 2}-\lambda^{\prime} \nu^{\prime}+2 \frac{\nu^{\prime}-\lambda^{\prime}}{r}\right)\right\}, \\
\frac{\left(\rho+P_{r}\right) \omega e^{(\nu+\lambda) / 2}}{1-\omega^{2}}=-\frac{\dot{\lambda}}{8 \pi r} .
\end{gathered}
$$

At the outside of the fluid distribution, the spacetime is that of Schwarzschild, given by

$$
d s^{2}=\left(1-\frac{2 M}{r}\right) d t^{2}-\left(1-\frac{2 M}{r}\right)^{-1} d r^{2}-r^{2}\left(d \theta^{2}+\sin ^{2} \theta d \phi^{2}\right)
$$

As is well known, in order to match smoothly the two metrics above on the boundary surface $r=r_{\Sigma}(t)$, we must require the continuity of the first and the second fundamental form across that surface. In our notation this implies

$$
\begin{gathered}
e^{\nu_{\Sigma}}=1-\frac{2 M}{r_{\Sigma}} \\
e^{-\lambda_{\Sigma}}=1-\frac{2 M}{r_{\Sigma}} .
\end{gathered}
$$

and

$$
\left[P_{r}\right]_{\Sigma}=0
$$


Where, from now on, subscript $\Sigma$ indicates that the quantity is evaluated at the boundary surface $\Sigma$.

Eqs. (20), (21) and (22) are the necessary and sufficient conditions for a smooth matching of the two metrics (4) and (19) on $\Sigma$.

Next, let us write the energy momentum tensor in the form

$$
T_{\mu \nu}=\left(\rho+P_{\perp}\right) u_{\mu} u_{\nu}-P_{\perp} g_{\mu \nu}+\left(P_{r}-P_{\perp}\right) s_{\mu} s_{\nu}
$$

with

$$
\begin{aligned}
& u^{\mu}=\left(\frac{e^{-\nu / 2}}{\left(1-\omega^{2}\right)^{1 / 2}}, \frac{\omega e^{-\lambda / 2}}{\left(1-\omega^{2}\right)^{1 / 2}}, 0,0\right), \\
& s^{\mu}=\left(\frac{\omega e^{-\nu / 2}}{\left(1-\omega^{2}\right)^{1 / 2}}, \frac{e^{-\lambda / 2}}{\left(1-\omega^{2}\right)^{1 / 2}}, 0,0\right),
\end{aligned}
$$

where $u^{\mu}$ denotes the four velocity of the fluid and $s^{\mu}$ is a radially directed space-like vector orthogonal to $u^{\mu}$. Then the radial component of the conservation law

$$
T_{\nu ; \mu}^{\mu}=0
$$

may be written as

$$
\left(-8 \pi T_{1}^{1}\right)^{\prime}=\frac{16 \pi}{r}\left(T_{1}^{1}-T_{2}^{2}\right)+4 \pi \nu^{\prime}\left(T_{1}^{1}-T_{0}^{0}\right)+\frac{e^{-\nu}}{r}\left(\ddot{\lambda}+\frac{\dot{\lambda}^{2}}{2}-\frac{\dot{\lambda} \dot{\nu}}{2}\right),
$$

which in the static case becomes

$$
P_{r}^{\prime}=-\frac{\nu^{\prime}}{2}\left(\rho+P_{r}\right)+\frac{2\left(P_{\perp}-P_{r}\right)}{r},
$$

representing the generalization of the Tolman-Oppenheimer-Volkof equation for anisotropic fluids [2]. Thus, as mentioned before, local anisotropy introduces an extra term in this "force" equation, besides the usual pressure gradient term.

Finally, for the two non-vanishing components of the four acceleration, we easily find

$$
a_{0}=\frac{1}{1-\omega^{2}}\left[\left(\frac{\omega \dot{\omega}}{1-\omega^{2}}+\frac{\omega^{2} \dot{\lambda}}{2}\right)+e^{\nu / 2} e^{-\lambda / 2}\left(\frac{\omega \nu^{\prime}}{2}+\frac{\omega^{2} \omega^{\prime}}{1-\omega^{2}}\right)\right],
$$




$$
a_{1}=-\frac{1}{1-\omega^{2}}\left[\left(\frac{\omega \omega^{\prime}}{1-\omega^{2}}+\frac{\nu^{\prime}}{2}\right)+e^{-\nu / 2} e^{\lambda / 2}\left(\frac{\omega \dot{\lambda}}{2}+\frac{\dot{\omega}}{1-\omega^{2}}\right)\right],
$$

\section{Integrating the Geodesic Condition}

Let us now integrate the geodesic condition. First, observe that from the field equations (15),(16) and (18), one obtains after simple manipulations

$$
\omega e^{(\nu-\lambda) / 2}\left(\lambda^{\prime}+\nu^{\prime}\right)+\left(1+\omega^{2}\right) \dot{\lambda}=0
$$

Next, it follows at once from (29) and (30) that,

$$
\omega a_{1}=-a_{0} e^{(\lambda-\nu) / 2}
$$

Therefore the vanishing four-acceleration condition amounts to

$$
\left(\frac{\omega \omega^{\prime}}{1-\omega^{2}}+\frac{\nu^{\prime}}{2}\right)+e^{-\nu / 2} e^{\lambda / 2}\left(\frac{\omega \dot{\lambda}}{2}+\frac{\dot{\omega}}{1-\omega^{2}}\right)=0 .
$$

Then, replacing $\nu^{\prime}$ by its expression from (31), into (33), this last equation becomes

$$
\omega e^{(\nu-\lambda) / 2}\left(\lambda^{\prime}-\frac{2 \omega \omega^{\prime}}{1-\omega^{2}}\right)+\dot{\lambda}-\frac{2 \omega \dot{\omega}}{1-\omega^{2}}=0
$$

or, using (14)

$$
\dot{\phi} d t+\phi^{\prime} d r=0
$$

whose solution is

$$
\phi=\ln \left(1-\omega^{2}\right)+\lambda=\text { Constant } .
$$

Finally, from the fact that $\omega(t, 0)=0$ we obtain,

$$
e^{-\lambda}=1-\omega^{2} \text {. }
$$

Introducing the mass function as usually,

$$
e^{-\lambda}=1-\frac{2 m}{r}
$$


we have

$$
m=\frac{\omega^{2} r}{2}
$$

In all the above we have assumed $\omega \neq 0$, since from simple physical considerations we should not expect static solutions to exist.

Indeed, if we assume staticity $(\omega=0)$ then the geodesic condition implies $\nu^{\prime}=0$, which in turn, using (16) and (38), leads to

$$
8 \pi P_{r}=-\frac{2 m}{r^{3}}
$$

Then junction condition (22) would lead to $m_{\Sigma}=M=0$.

There is however one possible case of static geodesic solution, which appears if we relax the condition of continuity of the second fundamental form (implying the continuity of radial pressure) across the boundary surface, and assume the existence of a surface layer [7].

In this specific case, it follows from (40), the geodesic condition and field equations (15) and (17) that

$$
\rho+P_{r}+2 P_{\perp}=0
$$

implying that the active gravitational mass (Tolman, [8]) defined for any $r<r_{\Sigma}$ as,

$$
m_{T}=4 \pi \int_{0}^{r} r^{2} e^{(\nu+\lambda) / 2}\left(T_{0}^{0}-T_{1}^{1}-2 T_{2}^{2}\right) d r
$$

vanishes inside the sphere.

We shall not consider here these kind of solutions and accordingly all our models will be dynamic $(\omega \neq 0)$ and satisfy all junction conditions .

Now, from (14) evaluated at the boundary surface, and (20), (21), we obtain

$$
\omega_{\Sigma}=\frac{\dot{r}_{\Sigma}}{1-2 M / r_{\Sigma}}
$$

On the other hand, from (39) evaluated at the boundary surface, we have

$$
\omega_{\Sigma}= \pm \sqrt{\frac{2 M}{r_{\Sigma}}}
$$


Where the $+(-)$ refers to the expansion (contraction) of the surface (from now on we shall only consider the contracting case). Feeding back (44) into (43), we get

$$
\omega_{\Sigma}=\frac{\dot{r}_{\Sigma}}{1-\omega_{\Sigma}^{2}}
$$

Then equating (45) and (44) we have

$$
\dot{r}_{\Sigma}=\left(\frac{2 M}{r_{\Sigma}}\right)^{3 / 2}-\left(\frac{2 M}{r_{\Sigma}}\right)^{1 / 2}
$$

This equation may be integrated to give

$$
\frac{t}{2 M}=2 \tanh ^{-1} \sqrt{\frac{2 M}{r_{\Sigma}}}-\frac{2\left[1+6 M / r_{\Sigma}\right]}{3\left(2 M / r_{\Sigma}\right)^{3 / 2}}
$$

giving the evolution of the boundary surface. Unfortunately this last equation cannot be inverted (at least we were unable to do that) to obtain the explicit form $r=r_{\Sigma}(t)$. Accordingly we have also integrated (46) numerically , in order to exhibit the evolution of $r_{\Sigma}$, see figure(1).

So far we have found all consequences derived from the geodesic condition which, obviously, are valid in the pure dust case as well as in the anisotropic case. In the next section we shall work out an explicit example by impossing an "equation of state" for the physical variables.

\section{A Model}

The purpose of this section is not to model any specific physical system, but just to illustrate the consequences derived from the geodesic condition. Thus, somehow inspired by the incompressible fluid model, let us assume

$$
T_{0}^{0}=f(t)
$$

then from (39) and the fact that

$$
m^{\prime}=4 \pi r^{2} T_{0}^{0}
$$

one obtains

$$
\omega=-r \sqrt{\frac{8 \pi}{3} f(t)}
$$


where

$$
f(t)=\frac{3 M}{4 \pi r_{\Sigma}^{3}} .
$$

Observe from (50) that the evolution in this model is homologous.

Next, introducing the dimensionless variables

$$
x \equiv \frac{r}{r_{\Sigma}} \quad ; \quad y \equiv \frac{r_{\Sigma}}{2 M}
$$

we have

$$
\begin{aligned}
e^{-\lambda} & =1-\frac{x^{2}}{y} \\
\omega & =-\frac{x}{\sqrt{y}}
\end{aligned}
$$

Finally, from the field equations the following relations follow

$$
\begin{gathered}
\rho y+P_{r} x^{2}=\frac{3\left(y-x^{2}\right)}{32 \pi M^{2} y^{3}} \\
P_{r} y+\rho x^{2}=\frac{\left(y-x^{2}\right)}{8 \pi}\left[\left(1-\frac{x^{2}}{y}\right)\left(\frac{1}{4 M^{2} x^{2} y^{2}}+\frac{\partial \nu}{\partial x} \frac{1}{4 M^{2} x y^{2}}\right)-\frac{1}{4 M^{2} x^{2} y^{2}}\right] \\
\rho+P_{r}=-\frac{3 \dot{y}\left(y-x^{2}\right)^{1 / 2} e^{-\nu / 2}}{8 \pi\left(2 M y^{3}\right)}
\end{gathered}
$$

Then from (55) and (56) we obtain $\rho+P_{r}$ as function of $\frac{\partial \nu}{\partial x}, x$ and $y$. Feeding back this expression into (57), this equation may be solved for $\nu$, which in turn allows to express all physical variables $\left(\rho, P_{r}\right.$ and $\left.P_{\perp}\right)$ in terms of $x$ and $y$ which are given by (47) or alternatively by the numerical solution of (46).

\section{Conclusions}

We have seen that the geodesic condition, which for anisotropic fluids is compatible with the presence of pressure gradients, can be integrated, giving the explicit form of the evolution of the boundary surface. Resulting models may be regarded as generalizations of Tolman-Bondi solutions, to anisotropic fluids. In order to obtain the evolution of all physical variables 
for different pieces of matter, additional information has to be given. In the model above we have assumed condition (48), which in turn leads to the homology condition (50). Parenthetically, this last condition is widely used by astrophysicists in their modelling of stellar structure and evolution [9].

Figures (1) and (2) display the behaviour of the radius and the evolution of $\omega_{\Sigma}$ in the contracting case. As expected, as the boundary surface approaches the horizon, its coordinate velocity $\left(\dot{r}_{\Sigma}\right)$ stalls, whereas the velocity $\omega_{\Sigma}$ measured by the locally Minkowskian observer, tends to light velocity. Figure (3) shows the sensitivity of the pattern evolution with respect to the compactness of the initial configuration. As expected more compact configurations collapse faster. The good behaviour of $\rho$ and $p_{r}$ is easily deduced from (55)-(57).

\section{Acknowledgements}

We acknowledge financial assistance under grant BFM2000-1322 (M.C.T. Spain) and from Cátedra-FONACIT, under grant 2001001789.

\section{References}

[1] G. Lemaitre, Ann. Soc. Sci. Bruxelles A53, 51 (1933).

[2] R. Bowers y E. Liang, Astrophys. J., 188, 657 (1974).

[3] L. Herrera and N. O. Santos, Phys. Rep. 286, 53 (1997); H. Bondi,Mon.Not.R.Astr.Soc. 262, 1088 (1993);L. Herrera, Phys. Lett. A 165, 206 (1992); W. Barreto, Astr.Space.Sci. 201, 191 (1993); A. Coley and B. Tupper, Class. Quantum Grav. 11, 2553 (1994); J. Martinez, D. Pavon and L. Nunez Mon.Not.R.Astr.Soc. 271, 463 (1994); T. Singh, P. Singh and A. Helmi, Il Nuov. Cimento 110B, 387 (1995); A. Das, N. Tariq and J. Biech, J.Math.Phys. 36, 340 (1995); R. Maartens, S. Maharaj and B. Tupper, Class.Quantum Grav. 12, 2577 (1995);G. Magli, Class. Quantum Grav. 14, 1937 (1997); A. Das, N. Tariq, D. Aruliah and T. Biech, J.Math.Phys. 38, 4202 (1997);L. Herrera, A. Di Prisco, J. Hernández-Pastora and N.O. Santos, Phys. Lett. A 237, 113 (1998); E. Corchero, Class.Quantum Grav. 15, 3645 (1998); E. Corchero, 
Astr.Space Sci. 259, 31 (1998); H. Bondi, Mon.Not.R.Astr.Soc. 302, 337 (1999); H. Hernandez, L. Nunez and U. Percoco, Class.Quantum Grav. 16, 897 (1999); T. Harko and M. Mark, J.Math. Phys. 41, 4752 (2000); A. Das and S. Kloster, Phys.Rev.D 62, 104002 (2000); 15, 3215; S. Jhingan and G. Magli Phys. Rev.D 61, 124006 (2000); L.Herrera, A. Di Prisco J. Ospino and E. Fuenmayor,J.Math.Phys. 42 2199 (2001); J. Krisch and E.Glass, J.Math.Phys. 43,1509 (2002); E. Corchero, Class.Quantum Grav. 19, 417 (2002);T. Harko and M. Mark, Ann. Phys. (Leipzig) 11, 3 (2002).

[4] R. Tolman, Proc.Nat.Acad.Sci. 20169 (1934);

H. Bondi, Mon.Not.R.Astr.Soc. 107410 (1947).

[5] J.R. Oppenheimer and H. Snyder, Phys. Rev. 56, 455, (1939).

[6] H. Bondi, Proc. R. Soc. A281, 39 (1964).

[7] W. Israel,Il Nuovo Cimento, 44B 1 (1966).

[8] R. Tolman, Phys. Rev., 35875 (1930).

[9] M. Schwarzschild, Structure and Evolution of the Stars, (Dover, New York) (1958); R. Kippenhahn and A. Weigert, Stellar Structure and Evolution, (Springer Verlag, Berlin) (1990); C. Hansen and S. Kawaler, Stellar Interiors: Physical principles, Structure and Evolution, (Springer Verlag, Berlin) (1994). 


\section{$7 \quad$ Figure captions}

- Figure 1. $y=r_{\Sigma} / 2 M$ as function of $t / M$ for the inital value $y(0)=30$.

- Figure 2. $\omega_{\Sigma}$ as function of $t / M$ for the same initial data as in figure 1.

- Figure 3. $\omega_{\Sigma}$ as function of $t / M$ for $y(0)=30,29,28,27,26$ curves from rigth to left respectively. 


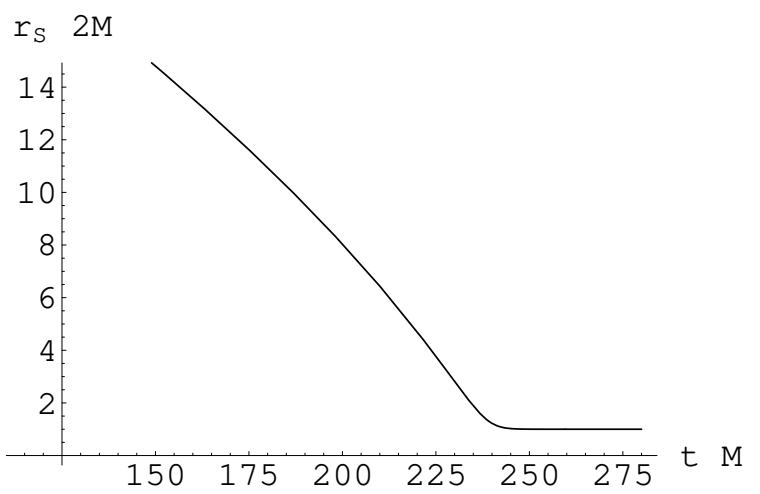

Figure 1.

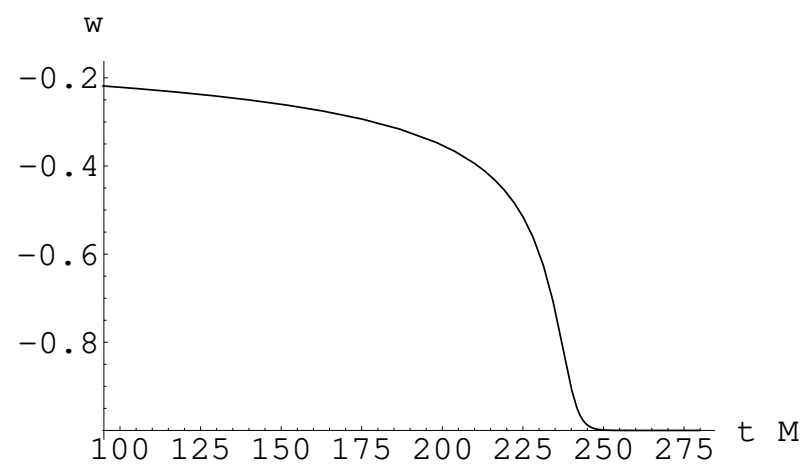

Figure 2 .

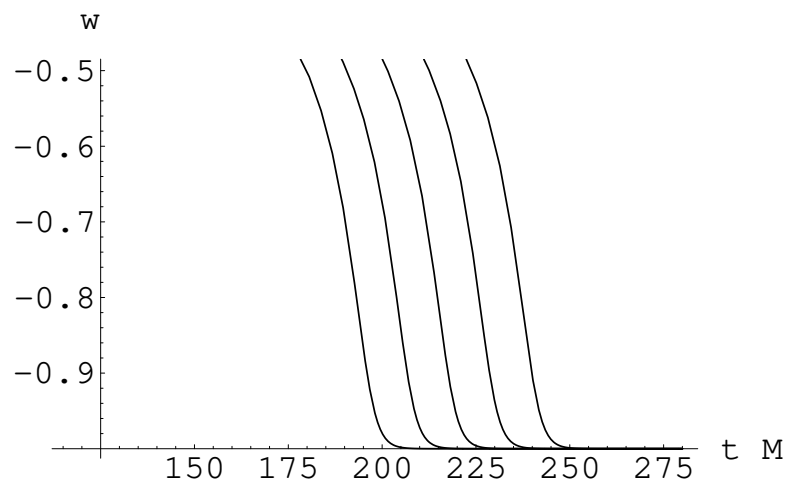

Figure 3. 